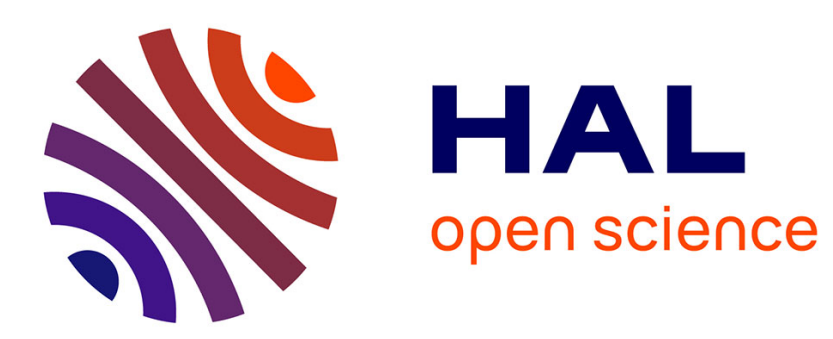

\title{
Détection et focalisation en milieu multi-cibles : méthode de la matrice de transfert
}

\author{
C. Prada, S. Manneville, M. Fink
}

\section{To cite this version:}

C. Prada, S. Manneville, M. Fink. Détection et focalisation en milieu multi-cibles: méthode de la matrice de transfert. Journal de Physique IV Proceedings, 1994, 04 (C5), pp.C5-1281-C5-1284. 10.1051/jp4:19945284 . jpa-00252996

\section{HAL Id: jpa-00252996 https://hal.science/jpa-00252996}

Submitted on 1 Jan 1994

HAL is a multi-disciplinary open access archive for the deposit and dissemination of scientific research documents, whether they are published or not. The documents may come from teaching and research institutions in France or abroad, or from public or private research centers.
L'archive ouverte pluridisciplinaire HAL, est destinée au dépôt et à la diffusion de documents scientifiques de niveau recherche, publiés ou non, émanant des établissements d'enseignement et de recherche français ou étrangers, des laboratoires publics ou privés. 


\title{
Détection et focalisation en milieu multi-cibles : méthode de la matrice de transfert
}

\author{
C. PRADA, S. MANNEVILLE et M. FINK
}

Laboratoire Ondes et Acoustique, Université Paris 7, ESPCI, 10 rue Vauquelin, 75005 Paris, France

\begin{abstract}
Résumé: Nous proposons une méthode de détection et de focalisation sélective en milieu multi-cibles. Elle est dérivée de l'analyse théorique du processus de retournement temporel itératif. Un réseau de transducteurs reversibles et un ensemble de cibles sont caractérisés par une matrice de transfert $\overline{\bar{K}}$, le processus de retournement temporel itératif est alors décrit par l'opérateur $\overline{\bar{K}}^{*} \overline{\bar{K}}$. On montre que pour des cibles ponctuelles et séparées par le réseau de transducteurs, chaque vecteur propre de $\overline{\bar{K}}^{*} \overline{\bar{K}}$, de valeur propre non nulle fournit la loi de focalisation sur l'une des cibles. Ces résultats sont validés expérimentalement.

Abstract: The iterative time reversal mirror is an efficient tool to focus among a set of targets on the most reflective one. This work provides a method to focus on the other targets. It is issued from the theoretical study of the iterative time reversal mirror. The transfer matrix of the system $\overline{\bar{K}}$ and the time reversal (TR) operator $\overline{\bar{K}}^{*} \overline{\bar{K}}$ are introduced. It is shown that for pointlike targets well resolved by the system, the phase and amplitude law to focus on any one of the targets is given by an eigenvector of the TR operator. The transfer matrix can easily be measured, then the diagonalization of the $\mathrm{TR}$ operator provides the number and position of the targets. This result is illustrated by an experimental example.
\end{abstract}

Introduction: La focalisation d'une onde acoustique sur une cible de position inconnue est un problème délicat. Le miroir à retournement temporel itératif apporte une solution élégante pour focaliser automatiquement dans un ensemble de cibles sur la plus réfléchissante d'entre elles. Ce système a des applications en contrôle non destructif, en lithotritic ou en acoustique sous-marine ${ }^{[1-3]}$. La question qui se pose alors naturellement est la suivante: comment focaliser sur les autres cibles? Ceci peut être intéressant en lithotritie lorsqu'il existe plusieurs lithiases, et plus généralement lorsqu'un réflecteur faible est masqué par un réflecteur fort. Une réponse a été trouvée dans l'étude théorique du processus de retournement temporel itératif. Cette étude est basée sur un formalisme matriciel: le réseau de $L$ transducteurs face à un milieu donné est considéré comme un système linéaire à $\mathrm{L}$ entrées et $\mathrm{L}$ sorties. On introduit alors la matrice de transfert du système ainsi que l'opérateur de retournement temporel. La théorie du retournement temporel itératif et la méthode de focalisation qui en découle sont exposées et illustrées par un exemple expérimental. 


\section{Théorie du miroir à retournement temporel itératif.}

Rappelons brièvement le principe du retournement temporel itératif. Le réseau de transducteurs émet une première onde incidente. L'onde réfléchie par la cible est captée puis réémise après retournement temporel. L'onde ainsi engendrée focalise sur la cible. Mais s'il existe plusieurs cibles, on peut réitérer cette opération plusieurs fois pour focaliser sur la plus réfléchissante: c'est le processus de retournement temporel itératif. Pour établir rigoureusement la convergence de ce processus une étude théorique est nécessaire. Une première approche a été présentée au deuxième congrès français d'acoustique ${ }^{[4]}$. Le point de vue adopté ici est un peu différent et apporte des éléments nouveaux qui dépassent l'étude de la convergence. Cette nouvelle approche repose sur la notion de matrice de transfert. ${ }^{[5]}$

- Matrice de transfert du système: Elle est définie à partir de la réponse impulsionnelle inter-élément $k_{l m}(t)$ de l'élément $l$ à l'élément $m . k_{l m}(t)$ est le signal reçu sur la voie $l$ lorsque la voie $m$ est soumise à une impulsion $\delta(t)$. L'acquisition de ces $L^{2}$ signaux peut se faire en excitant successivement les $L$ voies du réseau et en recevant l'écho du milieu simultanément sur toutes les voies. Le système étant supposé linéaire et invariant, les signaux $k_{l m}(t)$ pour $1 \leq l, m \leq L$ permettent de décrire toute opération d'émission-réception. En effet, si $e_{m}(t), 1 \leq m \leq L$, sont les $L$ signaux d'entrée, les $L$ signaux reçus $r_{l}(t), 1 \leq l \leq L$, sont donnés par

$$
r_{l}(t)=\sum_{m=1}^{L} k_{l m}(t) \underset{t}{\otimes e_{m}}(t), \quad 1 \leq l \leq L
$$

Ces équations se réduisent dans le domaine fréquentiel à l'équation matricielle suivante:

$$
\bar{R}(\omega)=\overline{\bar{I}}(\omega) \bar{E}(\omega)
$$

où $\bar{E}(\omega)=\left(E_{l}(\omega)\right)_{1 \leq l \leq L}$ et $\bar{R}(\omega)=\left(R_{l}(\omega)\right)_{1 \leq l \leq L}$ sont les vecteurs des transformées de Fourier des $L$ signaux émis et des $L$ signaux reçus, et où $\overline{\bar{K}}(\omega)=\left(K_{l m}(\omega)\right)_{1 \leq m, l \leq L}$ est la matrice de transfert du système. Dans la suite, la dépendance en $\omega$ est omise.

- Processus itératif: Le processus de retournement temporel itératif peut maintenant être décrit. Soit $\bar{E}^{0}$ le premier signal émis. Le signal reçu est alors $\bar{R}^{0}=\bar{K}_{\bar{E}}^{0}$. Le retournement temporel se traduisant par une conjugaison de phase dans le domaine fréquentiel, on démontre que les signaux émis à l'itération $2 n$ et à l'itération $2 n+1$ sont:

$$
\bar{E}^{2 n}=\left[\overline{\bar{K}}^{*} \overline{\bar{K}}\right]^{n} \bar{E}^{0} \text { et } \bar{E}^{2 n+1}=\left[\overline{\bar{K}}^{*} \overline{\bar{K}}^{n} \overline{\bar{K}}^{*} \bar{E}^{0 *}\right. \text {. }
$$

La matrice $\overline{\bar{K}}^{*} \overline{\bar{K}}$ est appelée opérateur de retournement temporel. On vérifie que $\overline{\bar{K}}^{*} \overline{\bar{K}}$ est hermitienne car le principe de réciprocité assure la symétrie de $\overline{\bar{K}}$. Ses valeurs propres sont réelles et même positives. Le comportement de $\left[\overline{\bar{K}}^{*} \overline{\bar{K}}\right]^{n}$ pour les grandes valeurs de $n$ peut donc être prévu. Soit $\lambda_{1}$ la plus grande valeur propre de $\overline{\bar{K}}^{*} \overline{\bar{K}}$ et $\bar{V}_{1}$ la projection de $\bar{E}_{0}$ sur l'espace propre associé à $\lambda_{1}$, on a alors

$$
\bar{E}^{2 n} \approx \lambda_{1}^{n} \bar{V}_{1}(n \rightarrow \infty)
$$

On vérifie que $\overline{\bar{K}}^{*} \bar{V}_{1}^{*}$ est aussi un vecteur propre associé à la valeur propre $\lambda_{1}$. En général cette valeur propre est non dégénérée et on montre que

$$
\bar{E}^{2 n+1} \approx \lambda_{1}^{n+1 / 2} e^{i \phi} \bar{V}_{1}(n \rightarrow \infty)
$$

Ceci assure la convergence du processus itératif vers une limite paire et une limite impaire qui diffèrent en général d'un terme de phase. 
- Détermination de la matrice de transfert: Pour D cibles ponctuelles en diffusion simple, $\overline{\bar{K}}$ est le produit de trois matrices: une matrice de propagation $\overline{\bar{H}}$, de dimension $D \times L$, qui relie les signaux émis par les transducteurs aux pressions reçues par les cibles, une matrice de diffusion $\overline{\bar{C}}$, de dimension $D \times D$, diagonale et dont les termes sont les coefficients de réflectivité des cibles, une matrice de propagation inverse qui d'après le principe de réciprocité est tout simplement ${ }^{t} \overline{\bar{H}}$. On a donc finalement

$$
\overline{\bar{K}}={ }^{t} \overline{\bar{H}} \overline{\bar{C}} \overline{\bar{H}}
$$

- Détermination des vecteurs propres: Si l'on fait l'hypothèse que les cibles sont idéalement résolues $(D<L)$ c'est à dire qu'il est possible de focaliser sur l'une quelconque des cibles en produisant une énergie nulle sur les autres, alors les vecteurs propres de l'opérateur de retournement temporel peuvent être déterminés. Notons $\bar{H}_{i}$ la ligne $i$ de la matrice $\overline{\bar{H}}$, c'est la réponse du diffuseur $i$ à l'ensemble des transducteurs. La focalisation optimale sur le diffuseur $i$ est obtenue par conjugaison de cette réponse c'est à dire en émettant le signal $\bar{H}_{i}^{*}$. On vérifie alors l'équation

$$
\overline{\bar{K}}^{*} \overline{\bar{K}} \bar{H}_{i}^{*}=\lambda_{i} \bar{H}_{i}^{*} \quad \operatorname{avec} \quad \lambda_{i}=\left|C_{i}\right|^{2}\left(\sum_{l=1}^{L}\left|H_{i l}\right|^{2}\right)^{2}
$$

Ceci détermine les $D$ valeurs propres non nulles de $\overline{\bar{K}}^{*} \overline{\bar{K}}$. Un vecteur propre de $\overline{\bar{K}}^{*} \overline{\bar{K}}$ de valeur propre non nulle fournit la loi de focalisation sur l'une des cibles. En particulier, le processus de retournement temporel itératif qui conduit à l'émission du vecteur propre de plus grande valeur propre, aboutit bien à une focalisation sur la cible la plus réfléchissante. L'hypothèse de résolution idéale est très restrictive, en pratique elle n'est pas bien satisfaite, mais cependant l'expérience montre que ce résultat reste valable si les cibles sont "assez espacées".

\section{Détection simultanée de plusieurs cibles.}

Des ces résultats découle la méthode de détection suivante: Pour un réseau de transducteurs placé face à un ensemble de cibles, les réponses impulsionnelles inter-éléments sont mesurées en excitant successivement chaque transducteur du réseau avec un même signal et en recevant l'écho sur toutes les voies. Par transformée de Fourier on calcule alors la matrice de transfert puis l'opérateur de retournement temporel. Ce dernier est diagonalisé à une fréquence choisie. Le nombre de cibles est alors le nombre de valeur propre non nulles. Leur position est donnée par les vecteurs propres.

- Résultats expérimentaux: Nous avons utilisé un réseau de 64 transducteurs de fréquence centrale $3 \mathrm{MHz}$, La cible est composée de deux fils distants de $3 \mathrm{~mm}$, placés perpendiculairement au réseau à une profondeur $\mathrm{z}=90 \mathrm{~mm}$. Afin de valider les résultats théoriques, nous avons comparé les résultats obtenus par retournement temporel et par le traitement de la matrice de transfert.

- Itération du RT: Deux itérations du processus de retournement temporel ont été faites. A chaque itération, le champ produit dans le plan des cibles a été mesuré avec un hydrophone déplacé pas à pas. A la première itération, on observe un lobe correspondant au fil le plus réflecteur et un deuxième lobe, d'amplitude 3 fois plus faible dû à l'autre fil. Ce deuxième lobe devient non significatif à la deuxième itération (Fig.1).

- Calcul et propagation des vecteurs propres principaux: Les réponses impulsionnelles inter-éléments de ce système ont été mesurées puis l'opérateur de retournement temporel calculé à la fréquence $3 \mathrm{MHz}$, a été diagonalisé. La distribution des valeurs propres met en évidence deux valeurs significatives (Fig.2). On émet successivement les vecteurs propres associés à ce deux valeurs et l'on mesure le champ dans le plan des cibles. L'émission du premier vecteur propre crée un lobe à l'emplacement du fil choisi par le processus de retournement temporel itératif. L'émission du deuxième vecteur propre crée un lobe à l'emplacement du deuxième fil (Fig.3). Ce résultat montre que cette méthode est valable même pour des cibles non idéalement résolues. 
Conclusion: On a montré comment la matrice de transfert d'un système constitué par un réseau de transducteurs et un ensemble de cibles pouvait être traitée pour extraire des informations sur le nombre et la position des cibles. On vérifie que lorsque les cibles sont résolues, chaque cible est associée à un vecteur propre qui donne une loi de focalisation sur celle-ci. Ainsi pour $N$ cibles ponctuelles, l'opérateur de retournement temporel a $N$ valeurs propres non nulles, les $N$ vecteurs propres associés sont les lois de focalisation sur chacunes des $\mathrm{N}$ cibles. De ce résultat découle une méthode de détection qui ne nécessite pas d'émetteur programmable mais requiert simplement l'acquisition de la matrice de transfert du système puis son traitement numérique. L'application de cette méthode à la détection et la focalisation en milieu inhomogène est à l'étude.

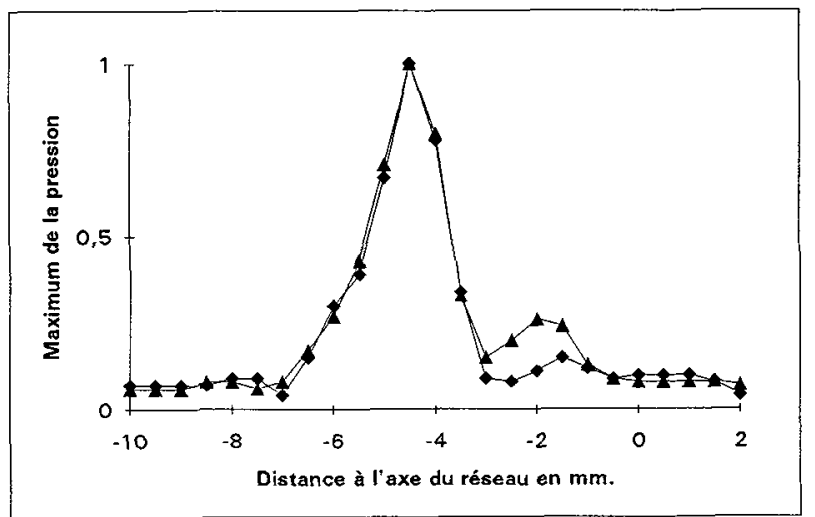

Figure 1:Diagramme de pression mesuré dans le plan des cibles à la première (triangles) et à la deuxième itération (losanges).

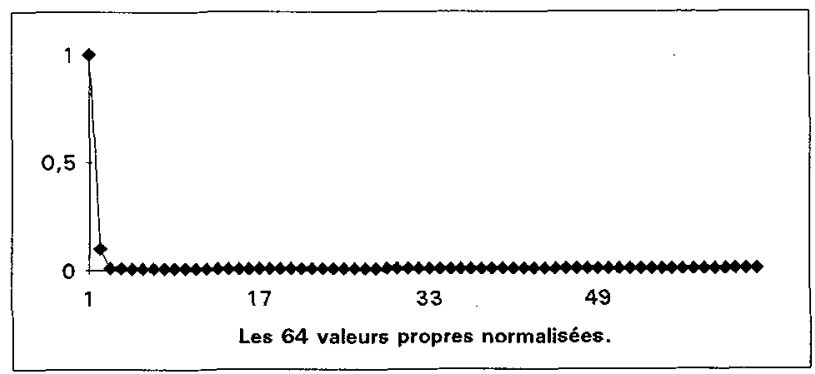

Figure 2: Distribution des valeurs propres.

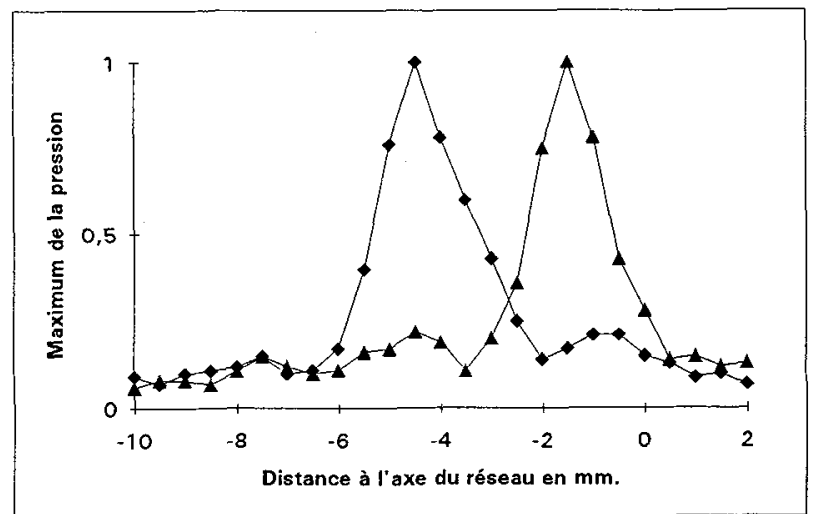

Figure 3: Diagramme de pression mesuré après émission du premier (losganges) et du deuxième (triangles) vecteur propre.

\section{Références}

[1] C. Prada, M. Fink, and F. Wu. "The iterative time reversal mirror: a solution to self focusing in pulse echo mode," J. Acoust. Soc. Am 1991 90(2) pp 1119-1129.

[2] C. Prada, "Retournement temporel des ondes ultra-sonores, application à la focalisation," Thèse de Doctorat, Juin 1991.

[3a] M. Fink, "Time Reversal of Ultrasonic Fields - Part I: Basic Principles,"

[3b] F. Wu, J.L. Thomas and M. Fink, "Time Reversal of Ultrasonic Fields - Part II: Experimental Results",

[3c] D. Cassereau and M. Fink, "Time Reversal of Ultrasonic Fields - Part III: Theory of the Closed Time-Reversal Cavity", IEEE Transactions on Ultrasonics, Ferroelectrics, and Frequency Control, (39), 5, 1992.

[4] C. Prada, N. Chakroun, J.L. Thomas, F. Wu et M. Fink, "Retournement temporel itératif: convergence du processus." 2ième CFA, Journal de Physique IV, Avril 92.

[5] C. Prada, J.L. Thomas, and M. Fink, "The iterative time reversal process: analysis of the convergence," Soumis à J. Acoust. Soc. Am. (Janvier 93). 